\title{
THE PSEUDOVARIETY OF ALL NILPOTENT GROUPS IS TAME
}

\author{
KHADIJEH ALIBABAEI \\ Centro de Matemática e Departamento de Matemática, Faculdade de \\ Ciências, Universidade do Porto, Porto, Portugal
}

\begin{abstract}
It has been shown that for every prime number $p$, the pseudovariety $\mathrm{G}_{p}$ of all finite $p$-groups is tame with respect to an implicit signature containing the canonical implicit signature. In this paper we generalize this result and we show that the pseudovariety of all finite nilpotent groups is tame but it is not completely tame.
\end{abstract}

Keywords. relatively free profinite semigroup, pseudovariety of semigroups, system of equations, implicit signature, completely tame, completely reducible, rational constraint, $\sigma$-full, weakly reducible.

\section{INTRODUCTION}

By a pseudovariety we mean a class of semigroups which is closed under taking subsemigroups, finite direct products, and homomorphic images. A pseudovariety is said to be decidable if there is an algorithm to test membership of a finite semigroup; otherwise, the pseudovariety is said to be undecidable. Eilenberg [14] established a correspondence between varieties of rational languages and pseudovarieties of finite semigroups which translates problems in language theory into the decidability of pseudovarieties of semigroups. In general the decidability of pseudovarieties is not preserved by many operations on pseudovarieties such as semidirect product, join and Mal'cev product $([19,1])$. Almeida and Steinberg introduced a refined version of decidability called tameness [11]. The tameness property requires the reducibility property which is a generalization of the notion of inevitability that Ash introduced to prove the type II conjecture of Rhodes [12].

There are various results using tameness of pseudovarieties to establish the decidability of pseudovarieties obtained by application of the operations of semidirect product, Mal'cev product and join [5, 4, 6].

Also there are connections between tameness and geometry and model theory [16, 15, 17, 8, 9]. So, it is worth finding more examples of tame pseudovarieties.

E-mail address: f.alibabaee@gmail.com.

2000 Mathematics Subject Classification. 20E18, 20M05, 20M07, 20F10, 20K01. 
It has been established that for every prime numbers $p$, the pseudovariety $\mathrm{G}_{p}$ of all finite $p$-groups is tame [3]. Using this result, in this paper we show that the pseudovariety $\mathrm{G}_{n i l}$ of all finite nilpotent groups is tame with respect to an enlarged implicit signature $\sigma$. Since the free $\sigma$-subalgebra generated by a finite alphabet $A$ is not any more the free group, in section 3, we prove the word problem is decidable in this free $\sigma$-subalgebra, meaning that there is an algorithm to decide whether two elements of this $\sigma$-algebra represent the same element.

In the last section, we show that the pseudovariety $\mathrm{G}_{n i l}$ is $\sigma$-reducible if and only of for all prime number $p$, the pseudovariety $\mathrm{G}_{p}$ is $\sigma$-reducible. This theorem yields as a corollary that the pseudovariety $\mathrm{G}_{n i l}$ is tame with respect to the systems of equations associated to finite directed graphs but is not completely tame.

\section{Preliminaries}

A topological semigroup is a semigroup $S$ endowed with a topology such that the basic semigroup multiplication $S \times S \rightarrow S$ is continuous. We say that a topological semigroup $S$ is A-generated if there is a mapping $\varphi: A \rightarrow S$ such that $\varphi(A)$ generates a dense subsemigroup of $S$.

A pseudovariety of semigroups is a class of finite semigroups closed under taking subsemigroups, homomorphic images, and direct products. Given a pseudovariety $\mathrm{V}$ of semigroups, by a pro- $\mathrm{V}$ semigroup $S$ we mean a compact, zero-dimensional semigroup which is residually in $\mathrm{V}$, that is for every two distinct points $s, t \in S$, there exists a continuous homomorphism $\varphi: S \rightarrow T$ into some member $T \in \mathrm{V}$ such that $\varphi(s) \neq \varphi(t)$.

For a finite set $A$ in the variety generated by $\mathrm{V}$, we denote by $\bar{\Omega}_{A} \mathrm{~V}$ the free pro- $\mathrm{V}$ semigroup. The free pro- $\mathrm{V}$ semigroup has the universal property in variety of pro- $\mathrm{V}$ semigroups in the sense that for every mapping $\varphi: A \rightarrow S$ into a pro- $\mathrm{V}$ semigroup $S$, there exists a unique continuous homomorphism $\hat{\varphi}: \bar{\Omega}_{A} \bigvee \rightarrow S$ such that the following diagram commutes:

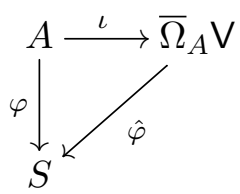

For an $A$-generated pro-V semigroup $S$, we view $S^{A}$ both as a direct power of $S$ and as the set of all functions from the set $A$ to $S$. To each element $w \in \bar{\Omega}_{A} \vee$, we may associate an $A$-ary operation $w_{S}: S^{A} \rightarrow S$ : for every $\varphi \in S^{A}$, by the universal property of $\bar{\Omega}_{A} \vee$, there is a unique extension $\hat{\varphi}$ : $\bar{\Omega}_{A} \bigvee \rightarrow S$. Define $w_{S}(\varphi)=\hat{\varphi}(w)$. It is easy to see that for every continuous homomorphism $f: S \rightarrow T$ between pro- $V$ semigroups, the following diagram 
commutes:

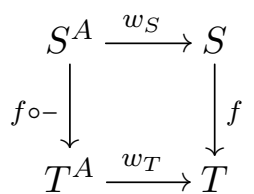

Operations with that property are called A-ary implicit operations. The element $w \in \bar{\Omega}_{A} \mathrm{~V}$ is completely determined by the implicit operation $\left(w_{S}\right)_{S \in \mathrm{V}}$ [3]. Note that, the elements of $A$ correspond to the component projects.

We say that an implicit operation $w \in \bar{\Omega}_{A} \mathrm{~V}$ is computable if there is an algorithm which given $S \in \mathrm{V}$ and $\varphi \in S^{A}$, output the value $w_{S}(\varphi)$.

An $A$-ary implicit operator on a pro-V semigroup $S$ is a transformation $f: S^{A} \rightarrow S^{A}$ of $S^{A}$ to itself whose components $f_{i}: S^{A} \rightarrow S$ determined an $A$-ary implicit operation. The set of all $A$-ary implicit operators on a pro-V semigroup $S$ is denoted by $\mathcal{O}_{A}(S)$. The set $\mathcal{O}_{A}(S)$ is a monoid under composition.

Proposition 2.1. [3, Proposition 2.2] There is a natural topology on $\mathcal{O}_{A}(S)$ such that the correspondence

$$
S \rightarrow \mathcal{O}_{A}(S)
$$

defines a functor from the category of pro- $\mathrm{V}$ semigroups with onto continuous homomorphisms as morphisms into the category of profinite monoids.

For $n$-ary implicit operations $w_{1}, \ldots, w_{n} \in \bar{\Omega}_{n} \vee$ and a pro- $\mathrm{V}$ semigroup $S$, denote by $\left(w_{1}, \ldots, w_{n}\right)$ the implicit operator

$$
\begin{aligned}
S^{n} & \rightarrow S^{n} \\
\left(s_{1}, \ldots, s_{n}\right) & \mapsto\left(\left(w_{1}\right)_{S}\left(s_{1}, \ldots, s_{n}\right), \ldots,\left(w_{n}\right)_{S}\left(s_{1}, \ldots, s_{n}\right)\right) .
\end{aligned}
$$

Denote composition of operators by concatenation: $\left(v_{1}, \ldots, v_{n}\right)\left(w_{1}, \ldots, w_{n}\right)$ has component $i$ determined by the operation $v_{i}\left(w_{1}, \ldots, w_{n}\right)$.

Recall that, for an element $v$ of a finite semigroup $V, v^{\omega}$ denotes the unique idempotent power of $v$. This defines a unary implicit operation $x \mapsto$ $x^{\omega}$ on finite semigroups (and similarly on finite monoids) which therefore has a natural interpretation on each profinite monoid of the form $\mathcal{O}_{n}(S)$. Note that $x^{\omega}$ is the limit of the sequence $\left\{x^{n !}\right\}_{n}$. We denote by $a_{j} \circ\left(w_{1}, \ldots, w_{n}\right)^{\omega}$, the $j$-th component of the $\omega$-power of the operator $\left(w_{1}, \ldots, w_{n}\right)$.

Lemma 2.2. [3, Corollary 2.5] Let $w_{1}, \ldots, w_{n} \in \bar{\Omega}_{n} \vee$. Then each component of $\left(w_{1}, \ldots, w_{n}\right)^{\omega}$ is also a member of $\bar{\Omega}_{n} \mathrm{~V}$. Moreover, if $w_{i}$ are computable operations, then so is each $a_{j} \circ\left(w_{1}, \ldots, w_{n}\right)^{\omega}$.

Let $\mathrm{S}$ be the pseudovariety of all finite semigroups. The elements of $\left(\bar{\Omega}_{A} \mathrm{~S}\right)^{1}$, over arbitrary finite alphabets $A$, are called pseudowords. A pseudoidentity is a formal equality of the form $u=v$ with $u, v \in \bar{\Omega}_{A} \mathrm{~S}$ for some finite alphabet $A$. For a pseudovariety $\mathrm{V}$ of semigroups, we denote by $\psi_{\mathrm{V}}$ the unique continuous homomorphism $\bar{\Omega}_{A} \mathrm{~S} \rightarrow \bar{\Omega}_{A} \mathrm{~V}$ which restricts to the identity on $A$. We say that $\mathrm{V}$ satisfies the pseudoidentity $u=v$ with $u, v \in \bar{\Omega}_{A} \mathrm{~S}$ if $\psi_{\mathrm{V}}(u)=\psi_{\mathrm{V}}(v)$. 
By an implicit signature we mean a set of pseudowords including multiplication. An important example is given by the canonical signature $\kappa$ consisting of the multiplication and the unary operation $x \mapsto x^{\omega-1}$ which, to an element $s$ of a finite semigroup with $n$ elements, associates the inverse of $s^{1+n !}$ in the cyclic subgroup generated by this power.

Let $\sigma$ be an implicit signature. Under the natural interpretation of the elements of $\sigma$, every profinite semigroup may be viewed as a $\sigma$-algebra in the sense of universal algebra. The $\sigma$-subalgebra of $\bar{\Omega}_{A} \vee$ generated by $A$ is denoted by $\Omega_{A}^{\sigma} \mathrm{V}$ and it is freely generated by $A$. We say that $\Omega_{A}^{\sigma} \mathrm{V}$ has decidable word problem if there is an algorithm to decide whether two pseudowords $u, v \in \bar{\Omega}_{A} \mathrm{~S}$ with $\psi_{\mathrm{V}}(u), \psi_{\mathrm{V}}(v) \in \Omega_{A}^{\sigma} \mathrm{V}$ represent the same implicit operation in $\Omega_{A}^{\sigma} \mathrm{V}$.

For every subset $L$ of $\bar{\Omega}_{A} \mathrm{~S}$ and $\mathrm{V}$ be a pseudovariety, we denote by $C l(L)$, $C l_{\mathrm{V}}(L)$, and $C l_{\sigma, \mathrm{V}}(L)$, the closure of $L$ in $\bar{\Omega}_{A} \mathrm{~S}, \bar{\Omega}_{A} \mathrm{~V}$, and $\Omega_{A}^{\sigma} \mathrm{V}$, respectively.

As it is mentioned in the introduction, the property tameness requires the property reducibility. To define reducibility we need a system of equations. Let $X$ and $P$ be disjoint finite sets, whose elements will be the variables and the parameters of the system, respectively. Consider the following system of equations:

$$
u_{i}=v_{i} \quad(i=1 \ldots, m),
$$

where $u_{i}$ and $v_{i}$ are pseudowords of $\bar{\Omega}_{X \cup P} S$. We also fix a finite set $A$ and for every $x \in X$, we choose a rational subset $L_{x} \subseteq A^{*}$. For every parameter $p \in P$, we associate an element $w_{p} \in \bar{\Omega}_{A} \mathrm{~S}$. A solution of the system (2.1) modulo $\mathrm{V}$ satisfying the constraints is a function $\delta: X \cup P \rightarrow \bar{\Omega}_{A} \mathrm{~S}$ satisfying the following conditions:

(1) $\delta(x) \in C l\left(L_{x}\right)$.

(2) $\delta(p)=w_{p}$.

(3) $\mathrm{V}$ satisfies the pseudoidentities $\delta\left(u_{i}\right)=\delta\left(v_{i}\right)(i=1 \ldots, m)$.

Theorem 2.3. [2, Theorem 5.6.1] Let $\mathrm{V}$ be a pseudovariety of finite semigroups. The following conditions are equivalent for a finite system $\Sigma$ of equations with rational constraints over the finite alphabet $A$ :

- $\Sigma$ has a solution modulo every A-generated semigroup in $\mathrm{V}$

- $\Sigma$ has a solution modulo $\bar{\Omega}_{A} \mathrm{~V}$.

Let $\sigma$ be an implicit signature. Consider a system of the form (2.1), with constraints $L_{x} \subseteq A^{*}$ and $w_{p} \in \Omega_{A}^{\sigma} \mathrm{S}(x \in X$ and $p \in P)$ where $u_{i}$ and $v_{i}$ are $\sigma$-terms in $\Omega_{X \cup P}^{\sigma} \mathrm{S}$. Assume that this system has a solution modulo $\mathrm{V}$. A pseudovariety $\mathrm{V}$ is said to be $\sigma$-reducible for this system if it has a solution $\delta: X \cup P \rightarrow \Omega_{A}^{\sigma} \mathrm{S}$ modulo $\mathrm{V}$. We say that $\mathrm{V}$ is completely $\sigma$-reducible if it is $\sigma$-reducible for every such system.

Proposition 2.4. [7, Proposition 3.1] Let $\mathrm{\vee}$ be a pseudovariety. If $\mathrm{\vee}$ is $\sigma$ reducible respect to the systems of equations of $\sigma$-terms without parameters, then $\mathrm{V}$ is completely $\sigma$-reducible. 
We say that a pseudovariety $\boldsymbol{V}$ is $\sigma$-tame with respect to a class $\mathfrak{C}$ of systems of equations if the following conditions hold:

- for every system of equations in $\mathfrak{C}$, the pseudovariety $\vee$ is $\sigma$-reducible;

- the word problem is decidable in $\Omega_{A}^{\sigma} \mathrm{V}$;

- $\mathrm{V}$ is recursively enumerable, in the sense that there is some Turing machine which outputs successively representatives of all the isomorphism classes of members of $V$.

We say that $\mathrm{V}$ is completely $\sigma$-tame if $V$ is completely $\sigma$-reducible. Some important tameness results are as follows:

- The pseudovariety $\mathrm{G}$ of all finite groups is $\kappa$-tame with respect to systems of equations associated with finite directed graphs [11, 12]. It follows from results of Coulbois and Khélif that $\mathrm{G}$ is not completely $\kappa$-tame [13.

- For a prime number $p$, the pseudovariety $\mathrm{G}_{p}$ of all finite $p$-groups is tame with respect to the systems of equations associated with finite directed graphs, but not $\kappa$-tame [3].

- The pseudovariety $\mathrm{Ab}$ of all finite abelian groups is completely $\kappa$ tame [10].

Consider a system of equations (2.1) with constraints $L_{x} \subseteq A^{*}$ and $w_{p} \epsilon$ $\Omega_{A}^{\sigma} \mathrm{S}(x \in X$ and $p \in P)$ where $u_{i}$ and $v_{i}$ are $\sigma$-terms in $\Omega_{X \cup P}^{\sigma} \mathrm{S}$. A pseudovariety $\mathrm{V}$ is said to be weakly $\sigma$-reducible with respect to this system if in the case it has a solution modulo $\mathrm{V}$, then there is a solution $\delta: X \cup P \rightarrow \bar{\Omega}_{A} \mathrm{~S}$ modulo $\mathrm{V}$ which satisfies the conditions $\psi_{\mathrm{V}}(\delta(x)) \in \Omega_{A}^{\sigma} \mathrm{V}(x \in X)$. It is obvious that if a pseudovariety $\mathrm{V}$ is $\sigma$-reducible, then it is weakly $\sigma$-reducible but the converse is not true. For a prime number $p$, the pseudovariety $\mathrm{G}_{p}$ of all finite $p$ groups is weakly $\kappa$-reducible but it is not $\kappa$-reducible [21, 11].

We say that a pseudovariety $\mathrm{V}$ is $\sigma$-full if for every rational language $L \subseteq A^{*}$, the set $\psi_{\vee}\left(C l_{\sigma}(L)\right)$ is closed in $\Omega_{A}^{\sigma} \bigvee$.

Proposition 2.5. [11, Proposition 4.5] Every $\sigma$-full weakly $\sigma$-reducible pseudovariety is $\sigma$-reducible.

Let $x \in \bar{\Omega}_{A} \mathrm{~S}$ and fix $n \in \mathbb{N}$. We denote by $x^{n^{\omega}}$, the pseudoword

$$
\lim _{k \rightarrow \infty} x^{n^{k !}} .
$$

Let $w_{1}, \ldots, w_{k}$ be group words. We denote by $M\left(w_{1}, \ldots, w_{k}\right)$ the $k \times|A|$ matrix whose $(i, j)$-entry is the sum of the exponents in the occurrences of the letter $a_{j} \in A$ in the word $w_{i}$.

Theorem 2.6. [3] Let $p$ be a prime number and $\sigma_{p}$ be the set of all implicit signature obtained by adding to the canonical signature $\kappa$ all implicit operations of the form

$$
a_{j} \circ\left(w_{1}, \ldots, w_{n}\right)^{\omega}
$$

with $j=1, \ldots, n, w_{i}$ are $\kappa$-terms such that the subgroup $\left\langle w_{1}, \ldots, w_{n}\right\rangle$ is $\mathrm{G}_{p^{-}}$ dense. Then $\mathrm{G}_{p}$ is $\sigma_{p}$-tame. 
We use the implicit operations in the preceding theorem and we prove the following theorem:

Theorem 2.7. Let $\sigma$ be the set of all implicit signature obtained by adding to the canonical signature $\kappa$ all implicit operations of the form

$$
\left(a_{j} \circ\left(w_{1}, \ldots, w_{n}\right)^{\omega}\right)^{m^{\omega}} \quad(m \in \mathbb{N})
$$

with $j=1, \ldots, n, w_{i}$ are $\kappa$-terms such that $\operatorname{det} M\left(w_{1}, \ldots, w_{n}\right) \neq 0$ and every prime number $p$ dividing $\operatorname{det} M\left(w_{1}, \ldots, w_{n}\right)$ also divides $m$. Then $\mathrm{G}_{n i l}$ is $\sigma$-tame.

Note that by [18, for a prime number $p$ and $\kappa$-words $w_{1}, \ldots, w_{n}$, the subgroup $\left\langle w_{1}, \ldots, w_{n}\right\rangle$ is $\mathrm{G}_{p}$-dense if and only if $\operatorname{det} M\left(w_{1}, \ldots, w_{n}\right) \neq 0(\bmod p)$

\section{The PSeudovariety $\mathrm{G}_{n i l}$ IS $\sigma$-TAme}

3.1. The word problem is decidable in $\Omega_{A}^{\sigma} \mathrm{G}_{n i l}$. We use the following lemmas to reduced the word problem in $\Omega_{A}^{\sigma} \mathrm{G}_{n i l}$ to the the word problem in the free group generated by $A$.

Lemma 3.1. Let $u, v \in \bar{\Omega}_{A} \mathrm{~S}$. Then the pseudoidentity $u=v$ is valid in $\mathrm{G}_{n i l}$ if and only if, for every prime number $p$, the pseudoidentity $u=v$ is valid in $\mathrm{G}_{p}$.

Proof. Since every p-group is a nilpotent group, if $\mathrm{G}_{n i l}$ satisfies the pseudoidentity $u=v$, then for every prime number $p, \mathrm{G}_{p}$ satisfies the pseudoidentity $u=v$.

The converse follows from fact that every finite nilpotent group is isomorphic to the direct product of its $p$-Sylow subgroups.

We denote by $\mathbb{P}$, the set of all prime numbers.

Lemma 3.2. Fix $p \in \mathbb{P}$. The pseudoidentity $x^{n^{\omega}}=x$ is valid in $\mathrm{G}_{p}$ if $n$ is not divisible by $p$ and the pseudoidentity $x^{n^{\omega}}=1$ is valid in $\mathrm{G}_{p}$ otherwise.

Proof. The result follows from the elementary Euler congruence theorem.

Theorem 3.3. For every pseudoword $u \in \Omega_{A}^{\sigma} S$, there is a computable cofinite subset $S(u)$ of $\mathbb{P}$ such that the following properties hold:

- there is a computable $\kappa$-word $w_{0}$ such that for every $p \in S(u)$, the pseudoidentity $u=w_{0}$ is valid in $\mathrm{G}_{p}$;

- for every $p_{i} \in\left\{p_{1}, \ldots, p_{r}\right\}=\mathbb{P} \backslash S(u)$, there is a computable $\kappa$-word $w_{i}$ such that the pseudoidentity $u=w_{i}$ is valid in $\mathrm{G}_{p_{i}}(1 \leq i \leq r)$.

In particular, for every prime number $p$, we have $\Omega_{A}^{\sigma} G_{p}=\Omega_{A}^{\kappa} G_{p}$.

Proof. Since every $u \in \Omega_{A}^{\sigma} \mathrm{S}$ is constructed from the letters in $A$ using a finite number of times the operations in $\sigma$ and the intersection of a finite number of cofinite sets is cofinite, it is enough to show that the statement of the theorem holds for every pseudoword $u \in \sigma \backslash \kappa$. 
Consider the following pseudoword in $\sigma \backslash \kappa$

$$
u=\left(a_{j} \circ\left(w_{1}, \ldots, w_{n}\right)^{\omega}\right)^{m^{\omega}} \quad(m \in \mathbb{N})
$$

where $j=1, \ldots, n, w_{i}$ are $\kappa$-terms such that $\operatorname{det} M\left(w_{1}, \ldots, w_{n}\right) \neq 0$ and every prime number $p$ dividing $\operatorname{det} M\left(w_{1}, \ldots, w_{n}\right)$ also divides $m$. Let

$$
S(u)=\mathbb{P} \backslash\{p \mid p \text { divides } m\} .
$$

If $p$ lies in $S(u)$, then by [18, Corollary 3.3], the subgroup $\left\langle w_{1}, \ldots, w_{n}\right\rangle$ is $\mathrm{G}_{p}$-dense in $\bar{\Omega}_{A} \mathrm{G}_{p}$. Hence, by [3, Lemma 6.5 ], the following pseudoidentity holds in $\mathrm{G}_{p}$

Consider $w_{0}=a_{j}$.

$$
a_{j} \circ\left(w_{1}, \ldots, w_{n}\right)^{\omega}=a_{j} .
$$

Otherwise, $p$ divides $m$ and therefore by Lemma 3.2 , the pseudoidentity

$$
\left(a_{j} \circ\left(w_{1}, \ldots, w_{n}\right)^{\omega}\right)^{m^{\omega}}=1
$$

holds in $\mathrm{G}_{p}$.

Corollary 3.4. The word problem is decidable in $\Omega_{A}^{\sigma} \mathrm{G}_{n i l}$.

Proof. Let $u, v \in \Omega_{A}^{\sigma} \mathrm{S}$. Then by Lemma 3.1, the pseudoidentity $u=v$ holds in $\mathrm{G}_{n i l}$ if and only if for every prime number $p$, the pseudoidentity $u=v$ holds in $\mathrm{G}_{p}$.

Since $\mathrm{G}_{p}$ is a pseudovariety of finite groups, the pseudoidentity $u=v$ is valid in $\mathrm{G}_{p}$ if and only if the pseudoidentity $u v^{\omega-1}=1$ is valid in $\mathrm{G}_{p}$. Now the result follows from the preceding lemma and the fact that the word problem is decidable in the free group.

3.2. The pseudovariety $\mathrm{G}_{n i l}$ is $\sigma$-reducible. We denote by $F G(A)$, the free group over $A$ and for a finitely generated (f.g.) subgroup $H$ of a free group, denote by $\mathbb{P}(H)$, the set of all prime numbers $p$ such that $H$ is $\mathrm{G}_{p}$-closed.

Proposition 3.5. [18, proposition 4.3] Let $H$ be a f.g. subgroup of $F G(A)$. The set $\mathbb{P}(H)$ is a finite or a cofinite subset of $\mathbb{P}$, and it is effectively computable.

Lemma 3.6. Let $H$ be a f.g. subgroup of $F G(A)$. Then there is a cofinite subset $S(H)$ of $\mathbb{P}$ and a f.g. subgroup $K$ of $F G(A)$ such that for every $p \in S(H), C l_{\mathrm{G}_{p}, \kappa}(H)=K$.

Proof. Let $K_{1}, \ldots, K_{m}$ be the set of all overgroups of the subgroup $H$ (i.e., the automaton of $K_{i}$ is a quotient of the automaton of $H$ ). For every prime number $p$, there is $i(1 \leq i \leq m)$ such that $C l_{\mathrm{G}_{p}, \kappa}(H)=K_{i}$ [18. Since the number of overgroups of $H$ is a finite set, there is $K_{j}$ and an infinite subset $S$ of $\mathbb{P}$ such that for every $p \in S$, we have $C l_{\mathrm{G}_{p}, \kappa}(H)=K_{j}(1 \leq j \leq m)$. If $S$ is cofinite, then we are done. For every $p \in S$, we have

$$
K_{j} \subseteq C l_{\mathrm{G}_{p}, \kappa}\left(K_{j}\right) \subseteq C l_{\mathrm{G}_{p}, \kappa}\left(C l_{\mathrm{G}_{p}, \kappa}(H)\right)=C l_{\mathrm{G}_{p}, \kappa}(H)=K_{j}
$$

and hence, $S \subseteq \mathbb{P}\left(K_{j}\right)$. Therefore, by the preceding proposition, $\mathbb{P}\left(K_{j}\right)$ is a cofinite subset of $\mathbb{P}$. 
Suppose that there is an overgroup $L$ of $H$ properly contained in $K_{j}$ such that $\mathbb{P}(L)$ is a cofinite subset of $\mathbb{P}$. Then for every $p \in \mathbb{P}(L)$, we have

$$
C l_{\mathrm{G}_{p}, \kappa}(H) \subseteq C l_{\mathrm{G}_{p}, \kappa}(L)=L \nsubseteq K_{j} .
$$

Hence, the set of all prime numbers $q$ such that $C l_{\mathrm{G}_{q}, \kappa}(H)=K_{j}$ is contained in the set $\mathbb{P} \backslash \mathbb{P}(L)$. Since we assume that $\mathbb{P}(L)$ is a cofinite subset of $\mathbb{P}$, the set $\mathbb{P} \backslash \mathbb{P}(L)$ is a finite set which contradicts the choice of $K_{j}$.

So, for every overgroup $L$ of $H$ properly contained in $K_{j}, \mathbb{P}(L)$ is a finite set. Let $K=K_{j}$ and

$$
S(H)=\underset{L}{\mathbb{P}(K) \backslash \underset{\text { overgroup of } H}{ } \bigcup_{C \subset K}} \mathbb{P}(L) .
$$

As the number of over groups of $H$ is finite, $S(H)$ is a cofinite subset of $\mathbb{P}$. For every $p \in S(H)$, we have

$$
C l_{\mathrm{G}_{p}, \kappa}(H) \subseteq C l_{\mathrm{G}_{p}, \kappa}(K)=K,
$$

hence, $C l_{\mathrm{G}_{p}, \kappa}(H)$ is a $\mathrm{G}_{p}$-closed overgroup of $H$ contained in $K$, by the choice of $p$, it follows that $C l_{\mathrm{G}_{p}, \kappa}(H)=K$.

The following two propositions are the main tools to show that the pseudovariety $\mathrm{G}_{n i l}$ is $\sigma$-reducible.

Proposition 3.7. Let $H_{1}, \ldots, H_{t}$ be f.g. subgroups of the free group and fix the cofinite sets $S\left(H_{i}\right)$ and the subgroups $K_{i}$ as in the preceding lemma. Let $w \in K_{1} \ldots K_{t}$. Then there are $u_{i} \in \Omega_{A}^{\sigma} \mathrm{S} \cap C l\left(H_{i}\right)$ and cofinite subsets $S_{1}\left(H_{i}\right)$ of $\mathbb{P}$ contained in $S\left(H_{i}\right)$ such that, if $p \in \bigcap_{i=1}^{t} S_{1}\left(H_{i}\right)$, then the pseudoidentity $w=u_{1} \ldots u_{t}$ is valid in $\mathrm{G}_{p}$ and the pseudoidentity $u_{i}=1$ is valid in $\mathrm{G}_{p}$ otherwise.

Proof. Since, for every prime number $p, \mathrm{G}_{p}$ is an extension-closed pseudovariety, by [18, Proposition 2.10], there are $\kappa$-words $w_{1, i}, \ldots, w_{s_{i}, i}$ such that $K_{i}=\left\langle w_{1, i} \ldots, w_{s_{i}, i}\right\rangle$ and $r k\left(K_{i}\right) \leq r k\left(H_{i}\right)(1 \leq i \leq t)$.

Fix $q \in \bigcap_{i=1}^{t} S\left(H_{i}\right)$. As $K_{i}$ contains $H_{i}$, we can rewrite the generator $h$ of $H_{i}$ as a reduced word $h^{\prime}$ in terms of generators of $K_{i}$. Since $C l_{\mathrm{G}_{q}, \kappa}\left(H_{i}\right)=K_{i}$, by [18, Proposition 2.9] $H_{i}$ is $\mathrm{G}_{q}$-dense in the pro- $\mathrm{G}_{q}$ topology on $K_{i}$. Hence, by [3. Proposition 5.2], there is a subset $\left\{h_{1, i}^{\prime}, \ldots, h_{s_{i}, i}^{\prime}\right\}$ of generators of $H_{i}$ such that the subgroup $H_{i}^{\prime}$ generated by $\left\{h_{1, i}^{\prime}, \ldots, h_{s_{i}, i}^{\prime}\right\}$ is $\mathrm{G}_{q}$-dense in the pro- $\mathrm{G}_{q}$ topology on $K_{i}$. Let $M\left(h_{1, i}^{\prime}, \ldots, h_{s_{i}, i}^{\prime}\right)$ be the $s_{i} \times s_{i}$ matrix whose $(k, j)$ entry is the number of occurrences of $w_{j, i}$ in $h_{k, i}^{\prime}$. Then by [18, Corollary 3.3 , we have $\operatorname{det} M\left(h_{1, i}^{\prime}, \ldots, h_{s_{i}, i}^{\prime}\right) \neq \equiv 0(\bmod q)$ and also for every prime number $p$ not dividing $\operatorname{det} M\left(h_{1, i}^{\prime}, \ldots, h_{s_{i}, i}^{\prime}\right)$, the subgroup $H_{i}^{\prime}$ is $p$-dense in the pro- $\mathrm{G}_{p}$ topology on $K_{i}$. Since for every $p \in S\left(H_{i}\right), K_{i}$ is $\mathrm{G}_{p}$-closed, by [18, Proposition 2.9] for every $p \in S\left(H_{i}\right)$ not dividing $\operatorname{det} M\left(h_{1, i}^{\prime}, \ldots, h_{s_{i}, i}^{\prime}\right)$ we have $C l_{\mathrm{G}_{p}, \kappa}\left(H_{i}^{\prime}\right)=C l_{\mathrm{G}_{p}, K_{i}}\left(H_{i}^{\prime}\right)=K_{i}$, where $C l_{\mathrm{G}_{p}, K_{i}}\left(H_{i}^{\prime}\right)$ is the $p$-closure of $H_{i}^{\prime}$ in the pro- $\mathrm{G}_{p}$ topology on $K_{i}$. Hence, by the proof of [3, Theorem 
6.1], for every $p \in S\left(H_{i}\right)$ not dividing $\operatorname{det} M\left(h_{1, i}^{\prime}, \ldots, h_{s_{i}, i}^{\prime}\right)$, the following pseudoidentity holds in $\mathrm{G}_{p}$

$$
w_{j, i}=a_{j} \circ\left(h_{1, i}, \ldots, h_{s_{i}, i}\right)^{\omega}
$$

where $h_{k, i}$ corresponds to $h_{k, i}^{\prime}$ as a word in terms of the alphabet $A$. Note that $a_{j} \circ\left(h_{1, i}, \ldots, h_{s_{i}, i}\right)^{\omega}$ belongs to $C l\left(H_{i}\right) \subseteq \bar{\Omega}_{A} \mathrm{~S}$.

Let

$$
S_{1}\left(H_{i}\right)=S\left(H_{i}\right) \backslash\left\{p \mid \operatorname{det} M\left(h_{1, i}^{\prime}, \ldots, h_{s_{i}, i}^{\prime}\right) \equiv 0 \quad(\bmod p)\right\} .
$$

As det $M\left(h_{1, i}^{\prime}, \ldots, h_{s_{i}, i}^{\prime}\right) \neq 0$, the sets $S_{1}\left(H_{i}\right)$ are cofinite subsets of $\mathbb{P}$.

Let $P_{0}=\left\{p_{1}, \ldots, p_{r}\right\}=\mathbb{P} \backslash \cap_{i=1}^{r} S_{1}(H)$ and $n_{0}=p_{1} \ldots p_{r}$. Consider the following pseudowords:

$$
u_{j, i}=\left(a_{j} \circ\left(h_{1, i}, \ldots, h_{s_{i}, i}\right)^{\omega}\right)^{n_{0}{ }^{\omega}} \in \Omega_{A}^{\sigma} \mathrm{S} \cap C l\left(H_{i}\right) .
$$

If $p \in \mathbb{P} \backslash P_{0}$, by Lemma 3.2 , the following pseudoidentities are valid in $\mathrm{G}_{p}$ :

$$
u_{j, i}=\left(a_{j} \circ\left(h_{1, i}, \ldots, h_{s_{i}, i}\right)^{\omega}\right)^{n_{0}{ }^{\omega}}=a_{j} \circ\left(h_{1, i}, \ldots, h_{s_{i}, i}\right)^{\omega} \stackrel{\sqrt[3.1]{-}}{-} w_{j, i} .
$$

Otherwise, the following pseudoidentities are valid in $\mathrm{G}_{p}\left(p \in P_{0}\right)$ :

$$
u_{j, i}=\left(a_{j} \circ\left(h_{1, i}, \ldots, h_{s_{i}, i}\right)^{\omega}\right)^{n_{0} \omega}=1 .
$$

So, we showed that for every generator $w_{k, i}$ of $K_{i}$, there are $u_{k, i} \in \Omega_{A}^{\sigma} \mathrm{S} \cap$ $C l\left(H_{i}\right)$ such that, if $p \in \bigcap_{i=1}^{r} S_{1}\left(H_{i}\right)$, then the pseudoidentity $u_{k, i}=w_{k, i}$ is valid in $\mathrm{G}_{p}$ and the pseudoidentity $u_{k, i}=1$ is valid in $\mathrm{G}_{p}$ otherwise. Hence, every $w \in K_{i}$ has this property $(1 \leq i \leq t)$.

Let $w \in K_{1} \ldots K_{t}$. Then there are $w_{i} \in K_{i}$ such that $w=w_{1} \ldots w_{t}$. By the preceding paragraph, there are $u_{i} \in \Omega_{A}^{\sigma} \mathrm{S} \cap C l\left(H_{i}\right)$ such if $p \in \bigcap_{i=1}^{r} S_{1}\left(H_{i}\right)$, then the pseudoidentity $u_{i}=w_{i}$ is valid in $\mathrm{G}_{p}$ and the pseudoidentity $u_{i}=1$ is valid in $\mathrm{G}_{p}$ otherwise. Let $v=u_{1} \ldots u_{t} \in \Omega_{A}^{\sigma} \mathrm{S} \cap \mathrm{Cl}\left(H_{1} \ldots H_{t}\right)$.

If $p \in \bigcap_{i=1}^{r} S_{1}\left(H_{i}\right)$, then the pseudoidentity $v=w$ is valid in $\mathrm{G}_{p}$ and the pseudoidentity $v=1$ is valid in $\mathrm{G}_{p}$ otherwise.

Proposition 3.8. Let $H_{1}, \ldots, H_{t}$ be f.g. subgroups of the free group and fix $S_{1}\left(H_{i}\right)$ as in the preceding proposition. Then for every $p$ in the set

$$
\mathbb{P} \backslash \bigcap_{i=1}^{t} S_{1}\left(H_{i}\right)=\left\{p_{1} \ldots, p_{r}\right\}
$$

and every $w \in C l_{\mathrm{G}_{p}, \kappa}\left(H_{1} \ldots H_{t}\right)$, there are $u_{i} \in \Omega_{A}^{\sigma} \mathrm{S} \cap C l\left(H_{i}\right)$ such that the pseudoidentity $u_{i}=1$ is valid in $\mathrm{G}_{q}$ for every $q \in \mathbb{P} \backslash\{p\}$ and the pseudoidentity $u_{1} \ldots u_{t}=w$ is valid in $\mathrm{G}_{p}$.

Proof. By [20, Lemma 5.2], for every prime number $p$, we have

$$
C l_{\mathrm{G}_{p}, \kappa}\left(H_{1} \ldots H_{t}\right)=C l_{\mathrm{G}_{p}, \kappa}\left(H_{1}\right) \ldots C l_{\mathrm{G}_{p}, \kappa}\left(H_{t}\right) .
$$

Let $P_{0}=\left\{p_{1}, \ldots, p_{r}\right\}$ and $K_{p_{i}, j}=C l_{\mathrm{G}_{p_{i}}, \kappa}\left(H_{j}\right)(1 \leq i \leq r$ and $1 \leq j \leq t)$. There are $\kappa$-words $w_{1, p_{i}, j}, \ldots, w_{s_{p_{i}, j}, p_{i}, j}$ such that

$$
K_{p_{i}, j}=\left\langle w_{1, p_{i}, j}, \ldots, w_{s_{p_{i}, j}, i, j}\right\rangle .
$$


By the proof of [3, Theorem 6.1], there is a subset $\left\{h_{1, p_{i}, j}, \ldots, h_{s_{p_{i}, j}, p_{i}, j}\right\}$ of the generators of $H_{j}$ such that

$$
C l_{\mathrm{G}_{p_{i}}, \kappa}\left(\left\langle h_{1, p_{i}, j}, \ldots, h_{s_{p_{i}, j}, p_{i}, j}\right\rangle\right)=C l_{\mathrm{G}_{p_{i}}, \kappa}\left(H_{j}\right)=K_{p_{i}, j},
$$

and the pseudoidentity

$$
w_{k, p_{i}, j}=a_{k} \circ\left(h_{1, p_{i}, j}, \ldots, h_{s_{p_{i}, j}, p_{i}, j}\right)^{\omega} \quad\left(1 \leq k \leq s_{p_{i}, j}\right)
$$

is valid in $G_{p_{i}}$. We consider the following finite subsets of $\mathbb{P}$ :

$$
P_{i}=\bigcup_{j=1}^{t}\left\{p \mid p \text { divides } \operatorname{det} M\left(h_{1, p_{i}, j}^{\prime}, \ldots, h_{s_{p_{i}, j}, p_{i}, j}^{\prime}\right)\right\} \quad(1 \leq i \leq r)
$$

where $h_{k, p_{i}, j}^{\prime}$ is generator $h_{k, p_{i}, j}$ written in terms of generators of $K_{p_{i}, j}(1 \leq$ $\left.k \leq s_{p_{i}, j}\right)$. Note that, since $K_{p_{i}, j}$ is a $p_{i}$-closed subgroup and

$$
C l_{\mathrm{G}_{p_{i}}, \kappa}\left(\left\langle h_{1, p_{i}, j}, \ldots, h_{s_{p_{i}, j}, p_{i}, j}\right\rangle\right)=K_{p_{i}, j},
$$

by [18, Proposition 2.9] the subgroup $\left\langle h_{1, p_{i}, j}^{\prime}, \ldots, h_{s_{p_{i}, j}, p_{i}, j}^{\prime}\right\rangle$ is $p_{i}$-dense in the pro- $\mathrm{G}_{p_{i}}$ topology on $K_{p_{i}, j}$ and, therefore, $p_{i}$ does not belong to $P_{i}$. Consider the following natural numbers:

$$
\begin{aligned}
n_{0} & =p_{1} \ldots p_{r}, \\
n_{i} & =\prod_{j=0}^{r} \prod_{p \in P_{j} \backslash\left\{p_{i}\right\}} p \quad(1 \leq i \leq r) .
\end{aligned}
$$

The natural numbers $n_{i}(1 \leq i \leq r)$ satisfy the following properties:

(1) The prime number $p_{i}$ does not divide $n_{i}$.

(2) Fix $p_{i} \in P_{0}$. For every $p_{j} \in P_{0}(j \neq i), p_{j}$ divides $n_{i}$, because $p_{j}$ belongs to the set $P_{0} \backslash\left\{p_{i}\right\}$.

(3) Since we have $p_{i} \notin P_{i}$, every prime number $p \in P_{i}$ divides $n_{i}$.

(4) For every prime number $p$ in $\left(P_{1} \cup \ldots \cup P_{r}\right) \backslash P_{0}, p$ divides $n_{i}(1 \leq i \leq$ $r$ ), because there is $j$ such that $p \in P_{j}$ and since $p$ does not belong to $P_{0}, p$ is in $P_{j} \backslash\left\{p_{i}\right\}$.

For every $i, j$, and $k\left(1 \leq j \leq s_{p_{i}, j}, 1 \leq i \leq r\right.$, and $\left.1 \leq j \leq t\right)$, we let

$$
\begin{aligned}
u_{k, p_{i}, j} & =\left(a_{k} \circ\left(h_{1, p_{i}, j}, \ldots, h_{s_{p_{i}, j}, p_{i}, j}\right)^{\omega}\right)^{n_{i} \omega} \\
& \left(a_{k} \circ\left(h_{1, p_{i}, j}, \ldots, h_{s_{p_{i}, j}, p_{i}, j}\right)^{\omega}\right)^{(\omega-1)\left(p_{i} n_{i}\right)^{\omega}} .
\end{aligned}
$$

Note that $u_{k, p_{i}, j}$ belongs to $\Omega_{A}^{\sigma} \mathrm{S} \cap C l\left(H_{j}\right)$. We claim that the pseudoidentity $u_{k, p_{i}, j}=w_{k, p_{i}, j}$ is valid in $\mathrm{G}_{p_{i}}$ and for every $p \in \mathbb{P} \backslash\left\{p_{i}\right\}$ and the pseudoidentity $u_{k, p_{i}, j}=1$ is valid in $\mathrm{G}_{p}\left(1 \leq k \leq s_{p_{i}, j}\right.$ and $\left.1 \leq j \leq t\right)$.

It remains to establish the claim. Consider the following cases:

- Let $p=p_{i}$. By the property (1) of $n_{i}, p_{i}$ does not divides $n_{i}$. Hence, we have the following pseudoidentities in $\mathrm{G}_{p_{i}}$

$$
\begin{aligned}
u_{k, p_{i}, j}= & \left(a_{k} \circ\left(h_{1, p_{i}, j}, \ldots, h_{s_{p_{i}, j}, p_{i}, j}\right)^{\omega}\right)^{n_{i} \omega} \\
& \left(a_{k} \circ\left(h_{1, p_{i}, j}, \ldots, h_{s_{p_{i}, j}, p_{i}, j}\right)^{\omega}\right)^{(\omega-1)\left(p_{i} n_{i}\right)^{\omega}} \\
= & \left(a_{k} \circ\left(h_{1, p_{i}, j}, \ldots, h_{s_{p_{i}, j}, p_{i}, j}\right)^{\omega}\right) 1 \stackrel{3.3)}{-} w_{k, p_{i}, j} .
\end{aligned}
$$


- Consider either $p \in P_{0} \backslash\left\{p_{i}\right\}$ or $p \in\left(P_{1} \cup \ldots \cup P_{r}\right) \backslash P_{0}$. By the property (2) and (4) of $n_{i}, p$ divides $n_{i}$. Hence, we have the following pseudoidentities in $\mathrm{G}_{p}$

$$
\begin{aligned}
& \left(a_{k} \circ\left(h_{1, p_{i}, j}, \ldots, h_{s_{p_{i}, j}, p_{i}, j}\right)^{\omega}\right)^{n_{i}{ }^{\omega}}=1, \\
& \left(a_{k} \circ\left(h_{1, p_{i}, j}, \ldots, h_{s_{p_{i}, j}, p_{i}, j}\right)^{\omega}\right)^{(\omega-1)\left(p_{i} n_{i}\right)^{\omega}}=1 .
\end{aligned}
$$

So, the pseudoidentity $u_{k, p_{i}, j}=1$ is valid in $\mathrm{G}_{p}$.

- Consider $p \in \mathbb{P} \backslash\left(P_{0} \cup \ldots \cup P_{r}\right)$. By the choice of $n_{i}, p$ does not divide $p_{i} n_{i}$ and, therefore, does not divide $n_{i}$. Hence, we have the following pseudoidentities in $\mathrm{G}_{p}$

$$
\begin{aligned}
u_{k, p_{i}, j}= & \left(a_{k} \circ\left(h_{1, p_{i}, j}, \ldots, h_{s_{p_{i}, j}, p_{i}, j}\right)^{\omega}\right)^{n_{i}{ }^{\omega}} \\
& \left(a_{k} \circ\left(h_{1, p_{i}, j}, \ldots, h_{s_{p_{i}, j}, p_{i}, j}\right)^{\omega}\right)^{(\omega-1)\left(p_{i} n_{i}\right)^{\omega}} \\
= & \left(a_{k} \circ\left(h_{1, p_{i}, j}, \ldots, h_{s_{p_{i}, j}, p_{i}, j}\right)^{\omega}\right) \\
& \left(a_{k} \circ\left(h_{1, p_{i}, j}, \ldots, h_{s_{p_{i}, j}, p_{i}, j}\right)^{\omega}\right)^{\omega-1} \\
= & 1 .
\end{aligned}
$$

This completes the proof of the claim.

We showed for every generator $w_{k, p_{i}, j}$ of $C l_{\mathrm{G}_{p_{i}, \kappa}}\left(H_{j}\right)=K_{p_{i}, j}$, there are $u_{k, p_{i}, j} \in \Omega_{A}^{\sigma} \mathrm{S} \cap \mathrm{Cl}\left(H_{j}\right)$ such that the pseudoidentity $u_{k, p_{i}, j}=w_{k, p_{i}, j}$ is valid in $\mathrm{G}_{p_{i}}$ and the pseudoidentity $u_{k, p_{i}, j}=1$ is valid in $\mathrm{G}_{p}\left(p \in \mathbb{P} \backslash\left\{p_{i}\right\}\right)$. Hence, every $w \in C l_{\mathrm{G}_{i}, \kappa}\left(H_{j}\right)$ has this property.

Fix $p \in P_{0}$ and let $w \in C l_{\mathrm{G}_{p}, \kappa}\left(H_{1} \ldots H_{t}\right)$. By (3.2), there are $w_{j} \epsilon$ $C l_{\mathrm{G}_{p}, \kappa}\left(H_{j}\right)$ such that $w=w_{1} \ldots w_{t}$. By the preceding paragraph, there are $u_{j} \in \Omega_{A}^{\sigma} \mathrm{S} \cap \mathrm{Cl}\left(H_{j}\right)$ such that the pseudoidentity $u_{j}=w_{j}$ is valid in $\mathrm{G}_{p}$ and the pseudoidentity $u_{j}=1$ is valid in $\mathrm{G}_{q}(q \in \mathbb{P} \backslash\{p\})$. Let

$$
v=u_{1} \ldots u_{t} \in \Omega_{A}^{\sigma} \mathrm{S} \cap C l\left(H_{1} \ldots H_{t}\right) .
$$

Then the equality $v=w$ is valid in $\mathrm{G}_{p}$ and the equality $v=1$ is valid in $\mathrm{G}_{q}$ $(q \in \mathbb{P} \backslash\{p\})$.

Corollary 3.9. For every prime number $p$, the pseudovariety $\mathrm{G}_{p}$ is $\sigma$ reducible with respect to the systems of equations associated with finite directed graphs.

Proof. By Theorem 3.3, for a rational subset $L$ of $A^{\star}$ we have

$$
C l_{\mathrm{G}_{p}, \sigma}\left(\psi_{\mathrm{G}_{p}}(L)\right)=C l_{\mathrm{G}_{p}, \kappa}\left(\psi_{\mathrm{G}_{p}}(L)\right) .
$$

Since the pseudovariety $G_{p}$ is weakly $\kappa$-reducible with respect to the systems of equations associated with finite directed graphs [21, by Theorem 2.5 we just need to show that $\mathrm{G}_{p}$ is $\sigma$-full.

By definition of $\sigma$-full pseudovariety, it is enough to show that for a rational subset of $A^{*}$ :

$$
\psi_{\mathrm{G}_{p}}\left(C l_{\sigma}(L)\right) \subseteq C l_{\mathrm{G}_{p}, \kappa}\left(\psi_{\mathrm{G}_{p}}(L)\right)
$$


or equivalently, it is enough to show that

$$
w \in C l_{\mathrm{G}_{p}, \kappa}\left(\psi_{\mathrm{G}_{p}}(L)\right) \Rightarrow C l(L) \cap \Omega_{A}^{\sigma} \mathrm{S} \cap\left(\psi^{-1}(w)\right) \neq \varnothing
$$

The proof is similar to the proof of [3, Theorem 6.1]. Since any rational subset of the free semigroup can be obtained by taking a finite number of finite subsets of the free semigroup and applying the union, product and the plus operation $L \rightarrow L^{+}$a finite number of times, it is enough to show that these operations preserve this property. As it is mentioned in the proof of [3, Theorem 6.2], for the rational subsets $L$ and $K$, we have

(1) $C l_{\mathrm{G}_{p}, \kappa}\left(\psi_{\mathrm{G}_{p}}(L)\right)=\psi_{\mathrm{G}_{p}}(L)$ if $L$ is finite;

(2) $C l_{\mathrm{G}_{p}, \kappa}\left(\psi_{\mathrm{G}_{p}}(L K)\right)=C l_{\mathrm{G}_{p}, \kappa}\left(\psi_{\mathrm{G}_{p}}(L)\right) C l_{\mathrm{G}_{p}, \kappa}\left(\psi_{\mathrm{G}_{p}}(K)\right)$

(3) $C l_{\mathrm{G}_{p}, \kappa}\left(\psi_{\mathrm{G}_{p}}(L \cup K)\right)=C l_{\mathrm{G}_{p}, \kappa}\left(\psi_{\mathrm{G}_{p}}(L)\right) \cup C l_{\mathrm{G}_{p}, \kappa}\left(\psi_{\mathrm{G}_{p}}(L)\right)$

(4) $C l_{\mathrm{G}_{p}, \kappa}\left(\psi_{\mathrm{G}_{p}}(L)^{+}\right)=C l_{\mathrm{G}_{p}, \kappa}\left(\left\langle\psi_{\mathrm{G}_{p}}(L)\right\rangle\right)$

If a language $L$ is finite, then any $w$ in $C l_{\mathrm{G}_{p}, \kappa}\left(\psi_{\mathrm{G}_{p}}(L)\right)$ is also an element of $C l(L) \cap \Omega_{A}^{\sigma} \mathrm{S} \cap\left(\psi^{-1}(w)\right)$ and so finite languages satisfy (3.5). Suppose that $L_{1}$ and $L_{2}$ are rational subsets of $\Omega_{A} S$ satisfy Property (3.4). By property (3), at least one of the sets $C l\left(L_{1}\right) \cap \Omega_{A}^{\sigma} \mathrm{S} \cap\left(\psi^{-1}(w)\right)$ and $C l\left(L_{2}\right) \cap \Omega_{A}^{\sigma} \mathrm{S} \cap\left(\psi^{-1}(w)\right)$ is nonempty and, therefore, so is their union.

Let $u \in \psi_{\mathrm{G}_{p}}\left(C l\left(L_{1} L_{2}\right)\right) \cap \Omega_{A}^{\sigma} \mathrm{G}_{p}$. By property (2), there are

$$
u_{i} \in C l_{\kappa, \mathrm{G}_{p}}\left(\psi_{\mathrm{G}_{p}}\left(L_{i}\right)\right) \quad(i=1,2)
$$

such that $u=u_{1} u_{2}$. By the induction hypotheses, there are $w_{i} \in \psi_{\mathrm{G}_{p}}^{-1}\left(u_{i}\right) \cap$ $\Omega_{A}^{\sigma} \mathrm{S} \cap C l\left(L_{i}\right)$. Let $w=w_{1} w_{2} \in \Omega_{A}^{\sigma} \mathrm{S} \cap C l\left(L_{1}\right) C l\left(L_{2}\right) \subseteq \Omega_{A}^{\sigma} \mathrm{S} \cap C l\left(L_{1} L_{2}\right)$. Then we have

$$
\psi_{\mathrm{G}_{p}}(w)=\psi_{\mathrm{G}_{p}}\left(w_{1} w_{2}\right)=\psi_{\mathrm{G}_{p}}\left(w_{1}\right) \psi_{\mathrm{G}_{p}}\left(w_{2}\right)=u_{1} u_{2}=u .
$$

Thus, $w$ belongs to $\Omega_{A}^{\sigma} \mathrm{S} \cap C l\left(L_{1} L_{2}\right) \cap \psi_{\mathrm{G}_{p}}^{-1}(u)$.

Let $L$ be a rational subset satisfies the property (3.5). By [3, Lemma 6.6], the is a finite subset $Y$ of $\kappa$ words, such that $\langle L\rangle=\langle Y\rangle$ and $C l(Y) \subseteq C L(L)$. Hence, to show that a rational language of the form $L^{+}$satisfies $(3.5)$, it is enough to establish that, for any finite subset $Y$ of $\kappa$ words the following property holds:

$$
w \in C l_{\mathrm{G}_{p}, \kappa}(\langle Y\rangle) \Rightarrow C l\left(Y^{+}\right) \cap \Omega_{A}^{\sigma} \mathrm{S} \cap\left(\psi^{-1}(w)\right) \neq \varnothing
$$

The result follows from the Propositions 3.7 and 3.8 by $t=1$.

Theorem 3.10. Let $\mathcal{C}$ be a class of systems of equations. For every prime number $p$, the pseudovariety $\mathrm{G}_{p}$ is $\sigma$-reducible with respect to the systems of equations in $\mathcal{C}$ if and only if the pseudovariety $\mathrm{G}_{n i l}$ is $\sigma$-reducible with respect to the systems of equations in $\mathcal{C}$

Proof. Let $\mathrm{V}$ and $\mathrm{W}$ be pseudovarieties of semigroups such that $\mathrm{V} \subseteq \mathrm{W}$. If a pseudoidentity is valid in $W$, then it is valid in $V$. Hence, if a equation has solution $\delta$ modulo $\mathrm{W}$, then $\delta$ is a solution modulo $\mathrm{V}$.

Conversely, let $X$ be a finite set of variable and let $k$ be the cardinality of $X$. Consider a system of equations in $\mathcal{C}$ of the form

$$
u_{i}=v_{i} \quad(i=1, \ldots, m),
$$


with rational constraints $L_{x} \subseteq\left(\Omega_{A} S\right)^{1}$ and $u_{i}, v_{i} \in \Omega_{X}^{\sigma} \mathrm{S}$. Suppose that this system has the solution $\delta: X \rightarrow \bar{\Omega}_{A} \mathrm{~S}$ modulo $\mathrm{G}_{n i l}$. Since $L_{x}$ is a rational subset of $\left(\Omega_{A} S\right)^{1}, L_{x}$ is a finite union of sets of the form $R_{0}^{*} w_{0} R_{1}^{*} \ldots w_{t} R_{t}^{*}$, where $R_{i}$ are rational subsets of $\left(\Omega_{A} S\right)^{1}$ and $w_{i} \in\left(\Omega_{A} S\right)^{1}$. Hence, for every $x \in X$, there is a simple rational subset

$$
L_{x}^{\prime}=R_{0, x}^{*} u_{0, x} R_{1, x}^{*} \ldots u_{t_{x}-1, x} R_{t_{x}, x}^{*} \subseteq L_{x}
$$

such that $\delta(x) \in C l_{\mathrm{G}_{n i l}}\left(L_{x}^{\prime}\right)$. So, the system (3.6) with constraints $L_{x}^{\prime} \subseteq \Omega_{A} \mathrm{~S}$ has the solution $\delta$ modulo $\mathrm{G}_{n i l}$ and, therefore, for every prime number $p$, the system (3.6) with constraints $L_{x}^{\prime} \subseteq \Omega_{A} \mathrm{~S}$ has a solution modulo $\mathrm{G}_{p}$. We show that the system (3.6) with constraints $L_{x}^{\prime}(x \in X)$ has a solution $\delta^{\prime}: X \rightarrow \Omega_{A}^{\sigma} S$ modulo $\mathrm{G}_{\text {nil }}$.

By [3, Lemma 6.6], there are finite subsets $Y_{i, x}$ of $\Omega_{A}^{\kappa} \mathrm{S}$ such that

$$
C l\left(Y_{i, x}^{*}\right) \subseteq C l\left(R_{i, x}^{*}\right),
$$

and the subgroup generated by $Y_{i, x}$ is equal to the subgroup generated by $R_{i, x}$ in the free group $\left(x \in X\right.$ and $\left.1 \leq i \leq t_{x}\right)$. Hence, we have

$$
C l_{\mathrm{G}_{p}}\left(R_{0, x}^{*} u_{0, x} R_{1, x}^{*} \ldots u_{t_{x}-1, x} R_{t_{x}, x}^{*}\right)=C l_{\mathrm{G}_{p}}\left(Y_{0, x}^{*} u_{0, x} Y_{1, x}^{*} \ldots u_{t_{x}-1, x} Y_{t_{x}, x}^{*}\right) .
$$

Let $S_{1}\left(\left\langle Y_{i, x}\right\rangle\right)$ be as in Proposition 3.7 and $S=\bigcap_{x \in X} \bigcap_{i=1}^{t_{x}} S_{1}\left(\left\langle Y_{i, x}\right\rangle\right)$. Fix $q \in S$. Since the system (3.6) with constraints $L_{x}^{\prime} \subseteq \Omega_{A} S$ has a solution modulo $\mathrm{G}_{q}$ and $\mathrm{G}_{q}$ is $\sigma$-reducible, there is a solution $\delta_{0}: X \rightarrow \Omega_{A}^{\sigma} \mathrm{S}$ modulo $\mathrm{G}_{q}$. Hence, for every $x \in X, \delta_{0}(x)$ lies in $C l_{\mathrm{G}_{q}, \sigma}\left(L_{x}^{\prime}\right)=C l_{\mathrm{G}_{q}, \kappa}\left(L_{x}^{\prime}\right)$. For every $p \in S$, we have

$$
\begin{aligned}
C l_{\mathrm{G}_{p}, \kappa}\left(L_{x}^{\prime}\right) & =C l_{\mathrm{G}_{p}, \kappa}\left(R_{0, x}^{*} u_{0, x} R_{1, x}^{*} \ldots u_{t_{x}-1, x} R_{t_{x}, x}^{*}\right) \\
& =C l_{\mathrm{G}_{p}, \kappa}\left(R_{0, x}^{*}\right) u_{0, x} C l_{\mathrm{G}_{p}, \kappa}\left(R_{1, x}^{*}\right) \ldots u_{t_{x}-1, x} C l_{\mathrm{G}_{p}, \kappa}\left(R_{t_{x}, x}^{*}\right) \\
& =C l_{\mathrm{G}_{p}, \kappa}\left(\left\langle R_{0, x}\right\rangle\right) u_{0, x} C l_{\mathrm{G}_{p}, \kappa}\left(\left\langle R_{1, x}\right\rangle\right) \ldots u_{t_{x}-1, x} C l_{\mathrm{G}_{p}, \kappa}\left(\left\langle R_{t_{x}, x}\right\rangle\right) \\
& =C l_{\mathrm{G}_{p}, \kappa}\left(\left\langle Y_{0, x}\right\rangle\right) u_{0, x} C l_{\mathrm{G}_{p}, \kappa}\left(\left\langle Y_{1, x}\right\rangle\right) \ldots u_{t_{x}-1, x} C l_{\mathrm{G}_{p}, \kappa}\left(\left\langle Y_{t_{x}, x}\right\rangle\right) \\
& =C l_{\mathrm{G}_{q}, \kappa}\left(\left\langle Y_{0, x}\right\rangle\right) u_{0, x} C l_{\mathrm{G}_{q}, \kappa}\left(\left\langle Y_{1, x}\right\rangle\right) \ldots u_{t_{x}-1, x} C l_{\mathrm{G}_{q}, \kappa}\left(\left\langle Y_{t_{x}, x}\right\rangle\right) \\
& =C l_{\mathrm{G}_{q}, \kappa}\left(Y_{0, x}^{*} u_{0, x} Y_{1, x}^{*} \ldots u_{t_{x}-1, x} Y_{t_{x}, x}^{*}\right) \\
& =C l_{\mathrm{G}_{q}, \kappa}\left(L_{x}^{\prime}\right) .
\end{aligned}
$$

So, for every $p \in S, \delta_{0}(x)$ lies in $C l_{\mathrm{G}_{p}, \kappa}\left(L_{x}^{\prime}\right)$.

By Proposition 3.7, there are $v_{i, x} \in C l\left(Y_{i, x}^{*}\right) \cap \Omega_{A}^{\sigma} \mathrm{S}$ such that if $p \in \mathbb{P} \backslash S$, then the pseudoidentity $v_{i, x}=1$ is valid in $\mathrm{G}_{p}$ and the pseudoidentity

$$
v_{0, x} u_{0, x} v_{1, x} \ldots u_{t_{x}-1, x} v_{t_{x}, x}=\delta_{0}(x) .
$$

is valid in $\mathrm{G}_{p}$ otherwise $\left(x \in X\right.$ and $\left.1 \leq i \leq t_{x}\right)$. Note that since $C l\left(Y_{i, x}^{*}\right) \subseteq$ $C l\left(R_{i, x}^{*}\right)$, the pseudowords $v_{0, x} u_{0, x} v_{1, x} \ldots u_{t_{x}-1, x} v_{t_{x}, x}$ lie in $C l\left(L_{x}^{\prime}\right)$.

Let $P=\left\{p_{1}, \ldots, p_{r}\right\}=\mathbb{P} \backslash S$. For every $p_{j} \in P$, there is a solution $\delta_{j}: X \rightarrow$ $\Omega_{A}^{\sigma} \mathrm{S}$ modulo $\mathrm{G}_{p_{j}}$ such that for every $x \in X, \delta_{j}(x)$ lies in $C l_{\mathrm{G}_{p_{j}}, \kappa}\left(L_{x}^{\prime}\right)$. 
By Proposition 3.7, there are $v_{i, x, p_{j}} \in C l\left(Y_{i, x}^{*}\right) \cap \Omega_{A}^{\sigma} \mathrm{S}$ such that if $p=p_{j}$, then the equality

$$
v_{0, i, p_{j}} u_{0, x} v_{1, x, p_{j}} \ldots u_{t_{x}-1, x} v_{t_{x}, x, p_{j}}=\delta_{j}(x)
$$

holds in $\mathrm{G}_{p}$ and the equality $v_{i, x, p_{j}}=1$ is valid in $\mathrm{G}_{p}$ otherwise $(x \in X$, $\left.1 \leq i \leq t_{x}, 1 \leq j \leq r\right)$. Note that since $C l\left(Y_{i, x}^{*}\right) \subseteq C l\left(R_{i, x}^{*}\right)$, the pseudowords

$$
v_{0, x, p_{j}} u_{0, x} v_{1, x, p_{j}} \ldots u_{t_{x}-1, x} v_{t_{x}, x, p_{j}}
$$

lie in $C l\left(L_{x}^{\prime}\right)$.

We define the function $\delta^{\prime}: X \rightarrow \Omega_{A}^{\sigma} \mathrm{S}$ with

$$
\begin{gathered}
\delta^{\prime}(x)=\left(v_{0, x} v_{0, x, p_{1}} \ldots v_{0, x, p_{r}}\right) u_{0, x}\left(v_{1, x} v_{1, x, p_{1}} \ldots v_{1, x, p_{r}}\right) \\
u_{t_{x}-1, x}\left(v_{t_{x}, x} v_{t_{x}, x, p_{1}} \ldots v_{t_{x}, x, p_{r}}\right) .
\end{gathered}
$$

We claim that $\delta^{\prime}$ is a solution of the system (3.6) modulo $\mathrm{G}_{n i l}$.

It remains to establish the claim. Note that since a closure of a subsemigroup is again a subsemigroup, the pseudoword $v_{i, x} v_{i, x, p_{1}} \ldots v_{i, x, p_{r}}$ lies in $C l\left(Y_{i, x}^{*}\right)$ and so, $\delta^{\prime}(x)$ lies in $C l\left(L_{x}^{\prime}\right)$. We show that the pseudovariety $\mathrm{G}_{n i l}$ satisfies the pseudoidentities $\delta^{\prime}\left(u_{i}\right)=\delta^{\prime}\left(v_{i}\right)(1 \leq i \leq m)$. By Lemma 3.1 , it is enough to show that for every prime number $p$, the pseudovariety $\mathrm{G}_{p}$ satisfies the pseudoidentities $\delta^{\prime}\left(u_{i}\right)=\delta^{\prime}\left(v_{i}\right)(1 \leq i \leq m)$.

First we show that

(1) if $p \in S$, then for every $x \in X$ the pseudoidentity

$$
\delta^{\prime}(x)=\delta_{0}(x)
$$

is valid in $\mathrm{G}_{p}$;

(2) if $p=p_{m} \in \mathbb{P} \backslash S$, then for every $x \in X$ the pseudoidentity

$$
\delta^{\prime}(x)=\delta_{m}(x)
$$

is valid in $G_{p_{m}}$.

We consider the following cases:

- Let $p \in S$. Then for every $x \in X$, the following pseudoidentities hold in $\mathrm{G}_{p}$ :

$$
v_{i, x, p_{j}}=1 \quad\left(1 \leq i \leq t_{x} \text { and } 1 \leq j \leq r\right),
$$

Hence, the following pseudoidentities hold in $\mathrm{G}_{p}$ :

$$
\delta^{\prime}(x)=v_{0, x} u_{0, x} v_{1, x} u_{t_{x}-1, x} v_{t_{x}, x} \stackrel{3.8}{-} \delta_{0}(x) .
$$

- Let $p=p_{m} \in \mathbb{P} \backslash S$. Then the following pseudoidentities hold in $\mathrm{G}_{p}$ :

$$
v_{i, x, p_{j}}=v_{s, x}=1 \quad\left(1 \leq i, s \leq t_{x} \text { and } j \neq m\right) .
$$

Hence, the following pseudoidentities hold in $\mathrm{G}_{p_{m}}$ :

$$
\delta^{\prime}(x)=v_{0, x, p_{m}} u_{0, x} v_{1, x, p_{m}} u_{t_{x}-1, x} v_{t_{x}, x, p_{m}} \stackrel{[3.9]}{=} \delta_{m}(x) .
$$

Now we show that for every prime number $p$, the pseudovariety $\mathrm{G}_{p}$ satisfies the pseudoidentities $\delta^{\prime}\left(u_{i}\right)=\delta^{\prime}\left(v_{i}\right)(1 \leq i \leq m)$. We consider the two following cases: 
- Let $p \in S$. Since $\delta_{0}$ is the solution of the system (3.6) modulo $\mathrm{G}_{p}$, $\delta_{0}(x)=\delta^{\prime}(x)(x \in X)$, and $u_{i}, v_{i} \in \Omega_{X}^{\sigma} \mathrm{S}$, the pseudovariety $\mathrm{G}_{p}$ satisfies the pseudoidentities $\delta^{\prime}\left(u_{i}\right)=\delta^{\prime}\left(v_{i}\right)(1 \leq i \leq m)$.

- consider $p_{j} \in \mathbb{P} \backslash S$. Since $\delta_{j}$ is the solution of the system (3.6) modulo $\mathrm{G}_{p_{j}}, \delta_{j}(x)=\delta^{\prime}(x)(x \in X)$, and $u_{i}, v_{i} \in \Omega_{X}^{\sigma} \mathrm{S}$, the pseudovariety $\mathrm{G}_{p_{j}}$ satisfies the pseudoidentities $\delta^{\prime}\left(u_{i}\right)=\delta^{\prime}\left(v_{i}\right)(1 \leq i \leq m)$.

This proves the theorem.

Corollary 3.11. The pseudovariety $\mathrm{G}_{\text {nil }}$ is $\sigma$-reducible with respect to the systems of equations associated with finite directed graphs.

Proof. The result follows from Corollary 3.9 and the preceding theorem.

Corollary 3.12. The pseudovariety $\mathrm{G}_{n i l}$ is not completely $\sigma$-reducible.

Proof. By the preceding Theorem, it is enough to show that for some prime number $p$, the pseudovariety $\mathbf{G}_{p}$ is not completely $\sigma$-reducible. Let $p$ be a odd prime number, $A=\{a, b\}$ and consider the following equation

$$
\left[x^{2} a, y^{-1} z^{2} b y\right]=1
$$

It has been shown that the equation 3.12 does not have solution in the free group [13]. We consider the following constraints:

- $L_{x}=\{a\}^{*}$;

- $L_{z}=\{b\}^{*}$;

- $L_{y}=A^{*}$.

Let $p$ be an odd prime number. We find a solution of the equation (3.12) with the above constraints modulo $\mathrm{G}_{p}$. The pseudowords $x=\left(a^{\omega-1}\right)^{2^{\omega-1}}$, $z=\left(b^{\omega-1}\right)^{2^{\omega-1}}$ and $y=1$ are a solutions of this equation modulo $\mathrm{G}_{p}$ because the pseudoidentities $\left(a^{\omega-1}\right)^{2^{\omega}}=a^{\omega-1}$ and $\left(b^{\omega-1}\right)^{2^{\omega}}=b^{\omega-1}$ hold in $\mathrm{G}_{p}$.

Since $\mathrm{G}_{p}$ is $\sigma$-full, $\mathrm{G}_{p}$ is $\sigma$-reducible for the equation (3.12) if and only if it has a solution $\delta: \bar{\Omega}_{X} \mathrm{~S} \rightarrow \bar{\Omega}_{A} \mathrm{~S}$ such that $\psi_{\mathrm{G}_{p}}(\delta(u))=1$ and $\psi_{\mathrm{G}_{p}}(\delta(x)) \epsilon$ $\Omega_{A}^{\sigma} \mathrm{G}_{p}$. But as $\Omega_{A}^{\sigma} \mathrm{G}_{p}=\Omega_{A}^{\kappa} \mathrm{G}_{p}$, the equation 3.12 must have a solution in the free group which is contradiction.

\section{ACKNOWLEDGMENTS}

This work is part of the author's preparation of a doctoral thesis under the supervision of Prof. Jorge Almeida, whose advice is gratefully acknowledged.

It was partially supported by the FCT Docoral Grant with reference (SFRH/ BD/98202/2013). It was also partially supported by CMUP (UID /MAT/00144/ 2013), which is funded by FCT (Portugal) with national (MEC) and European structural funds (FEDER), under the partnership agreement PT2020.

\section{REFERENCES}

1. D. Albert, R. Baldinger, and J. Rhodes, Undecidability of the identity problem for finite semigroups, J. Symbolic Logic 57 (1992), 179-192. (Cited on page 1) 
2. J. Almeida, Finite semigroups and universal algebra, Series in Algebra, vol. 3, World Scientific Publishing Co., Inc., River Edge, NJ, 1994, Translated from the 1992 Portuguese original and revised by the author. (Cited on page 4)

3. Dynamics of implicit operations and tameness of pseudovarieties of groups, Trans. Amer. Math. Soc. 354 (2002), 387-411. (Cited on pages 2, 3, 5, 7, 8, 9, $10,12$. and 13 )

4. J. Almeida, A. Azevedo, and M. Zeitoun, Pseudovariety joins involving J-trivial semigroups, Internat. J. Algebra Comput. 9 (1999), 99-112. (Cited on page 1.)

5. J. Almeida, J. C. Costa, and M. L. Teixeira, Semidirect product with an ordercomputable pseudovariety and tameness, Semigroup Forum 81 (2010), 26-50. (Cited on page 1 .)

6. J. Almeida, J. C. Costa, and M. Zeitoun, Tameness of pseudovariety joins involving $R$, Monatsh. Math. 146 (2005), 89-111. (Cited on page 1)

7. Complete reducibility of systems of equations with respect to $R$, Port. Math. (N.S.) 64 (2007), 445-508. (Cited on page 4)

8. J. Almeida and M. Delgado, Sur certains systèmes d'équations avec contraintes dans un groupe libre, Portugal. Math. 56 (1999), 409-417. (Cited on page 1.)

9. _ Sur certains systèmes d'équations avec contraintes dans un groupe libreaddenda, Portugal. Math. 58 (2001), 379-387. (Cited on page 1.)

10. _ Tameness of the pseudovariety of abelian groups, Internat. J. Algebra Comput. 15 (2005), 327-338. (Cited on page 5)

11. J. Almeida and B. Steinberg, On the decidability of iterated semidirect products with applications to complexity, Proc. London Math. Soc. (3) 80 (2000), 50-74. (Cited on pages 1 and 5 )

12. C. J. Ash, Inevitable graphs: a proof of the type II conjecture and some related decision procedures, Internat. J. Algebra Comput. 1 (1991), 127-146. (Cited on pages 1 and 5 )

13. T. Coulbois and A. Khélif, Equations in free groups are not finitely approximable, Proc. Amer. Math. Soc. 127 (1999), 963-965. (Cited on pages 5 and 15.)

14. S. Eilenberg, Automata, languages and machines, vol. A, Academic Press, New York, 1974. (Cited on page 1.)

15. R. Gitik, On the profinite topology on negatively curved groups, J. Algebra 219 (1999), 80-86. (Cited on page 1)

16. R. Gitik and E. Rips, On separability properties of groups, Internat. J. Algebra Comput. 5 (1995), 703-717. (Cited on page 1)

17. B. Herwig and D. Lascar, Extending partial automorphisms and the profinite topology on free groups, Trans. Amer. Math. Soc. 352 (2000), 1985-2021. (Cited on page 1.)

18. S. Margolis, M. Sapir, and P. Weil, Closed subgroups in pro-V topologies and the extension problem for inverse automata, Internat. J. Algebra Comput. 11 (2001), 405-445. (Cited on pages 6, 7, 8, and 10)

19. J. Rhodes, Undecidability, automata and pseudovarieties of finite semigroups, Internat. J. Algebra Comput. 9 (1999), 455-473. (Cited on page1)

20. L. Ribes and P. A. Zalesskiu, The pro-p topology of a free group and algorithmic problems in semigroups, Internat. J. Algebra Comput. 4 (1994), 359-374. (Cited on page 9 )

21. B. Steinberg, Inevitable graphs and profinite topologies: some solutions to algorithmic problems in monoid and automata theory, stemming from group theory, Internat. J. Algebra Comput. 11 (2001), 25-71. (Cited on pages 5 and 11) 